\title{
TYPE Ia SUPERNOVAE RATES AND GALAXY CLUSTERING FROM THE CFHT SUPERNOVA LEGACY
} SURVEY

\author{
M. L. Graham ${ }^{1}$, C. J. Pritchet ${ }^{1}$, M. Sullivan ${ }^{2}$, S. D. J. Gwyn ${ }^{1,3}$, J. D. Neill ${ }^{1,4}$, E. Y. Hsiao ${ }^{1}$, P. Astier ${ }^{5}$, D. Balam ${ }^{1}$, \\ C. Balland ${ }^{5}$, S. Basa ${ }^{6}$, R. G. Carlberg ${ }^{2}$, A. Conley ${ }^{2}$, D. Fouchez ${ }^{7}$, J. GuY ${ }^{5}$, D. Hardin ${ }^{5}$, I. M. Hook ${ }^{8}$, D. A. Howell ${ }^{2}$, \\ R. Pain ${ }^{5}$, K. Perrett ${ }^{2}$, N. Reghault ${ }^{5}$, S. Baumont ${ }^{5}$, J. Le Du ${ }^{7}$, C. Lidman $^{9}$, S. Perlmutter ${ }^{10}$, P. Ripoche ${ }^{7}$, N. Suzuki ${ }^{10}$, \\ E. S. WALKER ${ }^{8}$, AND T. ZHANG ${ }^{6}$ \\ ${ }^{1}$ Department of Physics and Astronomy, University of Victoria, PO Box 3055 STN CSC, Victoria BC V8T 1M8, Canada \\ ${ }^{2}$ Department of Astronomy and Astrophysics, University of Toronto, 60 St. George Street, Toronto ON M5S 3H8, Canada \\ ${ }^{3}$ Canadian Astronomy Data Centre, NRC Herzberg Institute for Astrophysics, 5071 West Saanich Road, Victoria BC V9E 2E7, Canada \\ ${ }^{4}$ California Institute of Technology, E. California Blvd., Pasadena CA 91125, USA \\ ${ }^{5}$ LPNHE, CNRS-IN2P3 and Universités Paris VI \& VII, 4 place Jussieu, 75252 Paris Cedex 05, France \\ ${ }^{6}$ LAM, CNRS, BP8, Traverse du Siphon, 13376 Marseille Cedex 12, France \\ ${ }^{7}$ CPPM, CNRS-IN2P3 and Université Aix-Marseille II, Case 907, 13288 Marseille Cedex 9, France \\ ${ }^{8}$ University of Oxford Astrophysics, Denys Wilkinson Building, Keble Road, Oxford OX1 3RH, UK \\ ${ }^{9}$ ESO, Alonso de Cordova 3107, Vitacura, Casilla 19001, Santiago 19, Chile \\ ${ }^{10}$ Lawrence Berkeley National Laboratory, 1 Cyclotron Road, Berkeley CA 94720 , USA \\ Received 2007 July 31; accepted 2008 January 26; published 2008 March 10
}

\begin{abstract}
The Canada-France-Hawaii Telescope Supernova Legacy Survey (CFHT SNLS) has created a large homogeneous database of intermediate redshift $(0.2<z<1.0)$ type Ia supernovae (SNe Ia). The SNLS team has shown that correlations exist between SN Ia rates, properties, and host galaxy star-formation rates (SFRs). The SNLS SN Ia database has now been combined with a photometric redshift galaxy catalog and an optical galaxy cluster catalog to investigate the possible influence of galaxy clustering on the SN Ia rate, over and above the expected effect due to the dependence of SFR on clustering through the morphology-density relation. We identify three cluster SNe Ia, plus three additional possible cluster SNe Ia, and find the SN Ia rate per unit mass in clusters at intermediate redshifts is consistent with the rate per unit mass in field early-type galaxies and the SN Ia cluster rate from low-redshift cluster targeted surveys. We also find the number of SNe Ia in cluster environments to be within a factor of 2 of expectations from the two-component SN Ia rate model.
\end{abstract}

Key words: galaxies: clusters: general - supernovae: general

Online-only material: color figure

\section{INTRODUCTION}

The importance of understanding Type Ia supernovae (SNe Ia) has risen greatly in recent years because of their pivotal role in demonstrating the accelerated expansion of the universe (Riess et al. 1998; Perlmutter et al. 1999; Astier et al. 2006; Wood-Vasey et al. 2007). SNe Ia are generally agreed to be carbon-oxygen white dwarfs which have accreted sufficient mass from their companion star to initiate a thermonuclear explosion. Despite this consensus, several models exist for the companion type, the accretion process, the delay time between star formation and SN Ia explosion, and the explosion mechanism (Hillebrandt \& Niemeyer 2000; Höflich et al. 2003). Photometric and spectroscopic observations, and explosion simulations, are making inroads towards a coherent picture of SN Ia events (Mazzali et al. 2007). Another (albeit indirect) path of investigation is studying the SN Ia rate in different stellar populations.

Compilation of the Cappellaro et al. (1999) supernova catalog with infrared data led Mannucci et al. (2005) to conclude that

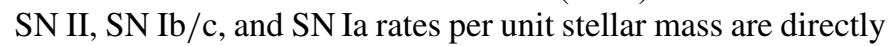
correlated with host morphology and $(B-K)$ color, and to infer a correlation with host star formation activity. Based on this, Scannapieco \& Bildsten (2005) expressed the SN Ia rate per unit stellar mass as the sum of a "delayed" component from old and a "prompt" component from young stellar populations. They parameterized the two components as A and B, proportional to the mass and star-formation rate (SFR) of a galaxy respectively, and also found the "prompt" component to account for the discrepancy between low observed rates of cluster SNe Ia and the high cluster iron abundance (Maoz \& Gal-Yam 2004). This " $A+B$ " two-component model has since been confirmed at intermediate redshifts with the large SNLS catalog (Sullivan et al. 2006a); the observations can also be matched with progenitor populations that have distributions of delay times as described by Mannucci et al. (2006) and Pritchet et al. (2008).

From the two-component model, the SN Ia rate in galaxy clusters is expected to be lower than in the field due to the morphology-density relation (Postman \& Geller 1984): cluster galaxies are predominantly of early type with little or no star formation. However, since clusters contain only a small fraction of the stellar mass of the universe, it is conceivable that some hitherto undetected influence in such exotic environments could affect the SN Ia rate. For example, the fraction of binary stars, or the rate of mass accretion onto the white dwarf, could be enhanced. The recent discovery of an enhanced nova rate in the core of elliptical galaxy M87 is evidence for the latter (Madrid et al. 2007). A second example is the detected SN Ia rate enhancement in radio-loud early-type galaxies (Della Valle et al. 2005); such galaxies tend to be very luminous ellipticals in the centers of large clusters.

Based on their analysis of two SN found in an archival survey of Hubble Space Telescope cluster images, Gal-Yam et al. (2002) find the SN Ia rate in cluster and field galaxies to be 
consistent at both low and high redshifts. The Wise Observatory Optical Transient Survey (WOOTS; Gal-Yam et al. 2008) targeting 140 Abell clusters confirmed the SNe Ia rate per unit mass in low-redshift galaxy clusters to be consistent with the rate in early-type galaxies (Sharon et al. 2007). Recently, Mannucci et al. (2007) analyzed the Cappellaro et al. (1999) sample of 136 low-redshift SNe $(\mathrm{z}<0.04)$ and found the SN Ia rate in cluster early-type galaxies is enhanced by a factor of $\gtrsim 3$ over field early-type galaxies. The large database of intermediate to high redshift SNe Ia compiled by the Canada-FranceHawaii Telescope Supernova Legacy Survey (CFHT SNLS) (Astier et al. 2006), and the publicly available photometric redshift galaxy and cluster catalogs for SNLS fields (Ilbert et al. 2006; Olsen et al. 2007) are ideal for extending these investigations to higher redshifts. As the SNLS is not a cluster-targeted survey, further advantages over Sharon et al. (2007) include using a flexible parametrization of galaxy clustering in the environments of SNe Ia, and determining simultaneous field SN Ia rates for comparison to cluster rates.

Section 2 describes the SN Ia, galaxy, and cluster catalogs used in this experiment. Sections 3 and 4 present two independent approaches to statistically evaluate the effects of clustering on the SN Ia rate per unit mass. Section 3 uses a continuous clustering strength estimator to compare the $\mathrm{SN}$ Ia rate per unit mass inside and outside clustered environments. Section 4 identifies six SNe Ia in Olsen et al. (2007) galaxy clusters, considers the probability of these observations based on expectations from the two-component rate model, and calculates the SN Ia rate per unit mass in clusters. Section 5 reviews and discusses the paper's main findings.

A flat cosmology with $H_{0}=70 \mathrm{~km} \mathrm{~s}^{-1} \mathrm{Mpc}^{-1}, \Omega_{\text {Lambda }}=$ 0.7 , and $\Omega_{M}=0.3$ is used.

\section{OBSERVATIONS}

Three catalogs are described here, all of which were generated from the four Deep fields of the CFHT Legacy Survey: the Supernova Legacy Survey catalog of type Ia supernovae ${ }^{11}$, the Ilbert et al. (2006) photometric redshift galaxy catalog, and the Olsen et al. (2007) optical galaxy cluster catalog.

\subsection{The CFHT Supernova Legacy Survey Catalog}

The SNLS images four $1 \mathrm{deg}^{2}$ Deep fields (D1-D4) every three to four nights (when visible) in four MegaCam filters $\left(g_{M}, r_{M}, i_{M}, z_{M}\right)$ to a depth $i_{M} \simeq 25$. At the end of its fiveyear program, the SNLS will have discovered $\sim 500$ type Ia supernova with its imaging and spectroscopic programs (Howell et al. 2005), and be a valuable contributor to collaborative efforts in constraining the dark energy equation-of-state parameter $w$ (Astier et al. 2006; Spergel et al. 2007). For this project we use the 343 SNe Ia spectroscopically identified prior to 2007 September 29.

\subsection{Ilbert Photometric Redshift Galaxy Catalog}

The Ilbert et al. (2006) galaxy photometric redshift catalog ${ }^{12}$ covers the four SNLS Deep fields. They incorporate VIMOS VLT Deep Survey (VVDS) spectroscopic redshifts to calibrate the spectral energy distribution (SED) fitting routine, resulting in photometric redshifts with an accuracy of $\sigma_{\Delta z /(1+z)}=0.029$ for $i_{A B}<24$, and a fraction of catastrophic errors of $1 \%$ at

\footnotetext{
11 http://legacy.astro.utoronto.ca.

12 http://terapix.iap.fr.
}

$17.5<i_{A B}<21.5$, increasing to $10 \%$ at $23.5<i_{A B}<24$ (Ilbert et al. 2006). In optimizing the photo- $z$ calculation, the accuracies of the SEDs types are compromised (Ilbert, private communication), and the distribution of SED types is discontinuous. To solve this problem, we use 51 SEDs interpolated from Coleman et al. (1980) and Kinney et al. (1996) templates ${ }^{13}$, and fit them to the apparent magnitudes and photometric redshifts of the catalog galaxies. We then calculate $K$ corrections and absolute magnitudes, and estimate galaxy stellar masses and star formation rates using fits of this library of SEDs to the models of Buzzoni et al. (2005), which we find to agree well (within a factor of 2) with those derived from PEGASE models (Sullivan et al. 2006b).

To ensure catalog purity, we restrict the catalog as recommended by Ilbert et al. (2006). For example, galaxies must be detected in $i_{M}$, the flag values are used to mask galaxies near foreground stars, the number of bands used for redshift fit must be at least 3, and galaxies with a second peak in their redshift probability distribution function indicate a likely catastrophic failure, thus are excluded (Ilbert et al. 2006). In addition, we impose the limits $i_{M}<25$ and $z<1.2$, and that the "object" parameter must be equal to zero indicating the object is a galaxy.

\subsection{Olsen Optical Galaxy Cluster Catalog}

The Olsen et al. (2007) cluster catalog derived from the Terapix data release in 2005 August (T0002) includes photometric redshifts and an optical grade (A-D). Olsen clusters are restricted to those of grade A, meaning a concentration of similarly colored galaxies is visible, resulting in $18,17,16$, and 10 clusters in D1-4. This optical cluster catalog was chosen over those of other selection techniques as it provides consistent completeness for all four SNLS Deep field areas.

Olsen et al. (2007) find their cluster redshifts are slightly overestimated for $z<0.6$, and underestimated for $z>0.7$, with a standard deviation of $\sim 0.1$ (Olsen et al. 2007). For the cluster redshift we instead use the peak of the redshift distribution from Ilbert catalog E/S0 and Sbc galaxies along the cluster's line of sight (within 40 arcsec). For distributions which plateau instead of peak, the plateau's central redshift is used. To evaluate corrected cluster redshift precision we apply the same procedure to the VVDS spectroscopic redshift catalog. ${ }^{14}$ For the four D1 clusters with VVDS galaxies along their line of sight, the differences between photometric and spectroscopic redshift peaks have a standard deviation of $\sigma_{\Delta z /(1+z \mathrm{vvDs})}=0.023$, indicating good agreement.

\subsection{Additional Catalog Processing}

$\mathrm{SNe}$ Ia host galaxies are identified as the closest neighbor in the Ilbert catalog, except when the offset difference between the nearest two is less than 2 arcsec, in which case redshift is used to discriminate. $85 \mathrm{SNe}$ Ia have no neighbor within 5 arcsec (maximum host offset), and cannot be used; this mainly includes SNe Ia in hosts of $i_{M}>25$, and on the masked regions covering $\sim 20 \%$ of field area. For the SNe Ia with potential Ilbert catalog hosts, iterative outlier rejection is applied to the residual dispersion between host photometric and SN Ia spectroscopic redshifts. ${ }^{15}$ The photometric redshift accuracy when performed for all fields combined is $\sigma_{\Delta z /\left(1+z_{\mathrm{SN}}\right)}=0.030$

\footnotetext{
13 http://www.astro.uvic.ca/ gwyn/cfhtls.

$14 \mathrm{http}: / /$ www.oamp.fr/virmos/.

15 A sigma of three and fractional convergence threshold of 0.01 was used; the average number of iterations was 4 .
} 
Table 1

SN Ia Rate Correction Factors

\begin{tabular}{lcccc}
\hline \hline Redshift Range & D1 & D2 & D3 & D4 \\
\hline $0.0-0.5$ & 1.431 & 1.016 & 1.538 & 0.896 \\
$0.5-0.7$ & 1.203 & 0.511 & 0.940 & 0.999 \\
$0.7-0.9$ & 0.523 & 0.194 & 0.418 & 0.616 \\
$0.9-1.1$ & 0.078 & 0.065 & 0.140 & 0.118 \\
$1.1-1.3$ & 0.000 & 0.000 & 0.000 & 0.000 \\
\hline
\end{tabular}

(consistent with Ilbert et al. 2006). However, if outlier rejection is applied to each Deep field separately, we find that each has a different photometric redshift uncertainty: $\sigma_{D 1}=0.028, \sigma_{D 2}=$ $0.024, \sigma_{D 3}=0.030, \sigma_{D 4}=0.050$. In this way, $26 \mathrm{SNe}$ Ia are rejected as outliers, dropping the total number to $232 \mathrm{SNe}$ Ia.

The SNIa rate per year, $\mathrm{SNR}_{\mathrm{Ia}}$, is calculated for every galaxy based on the two-component model, parameterized by $\mathrm{SNR}_{\mathrm{Ia}}=A M+B \dot{M}$, where $M$ is the stellar mass of the galaxy (in solar masses) and $\dot{M}$ is the star formation rate (in solar masses per year). The most recent $A$ and $B$ values of Sullivan et al. (2006a) are used: $A=5.3 \pm 1.1 \times 10^{-14} \mathrm{SNe} \mathrm{yr}^{-1} M_{\odot}^{-1}$ and $B=3.9 \pm 0.7 \times 10^{-4} \mathrm{SNe} \mathrm{yr}^{-1}\left(M_{\odot} \mathrm{yr}^{-1}\right)^{-1}$. The expected SN Ia rate per year for each galaxy is corrected to reproduce the total number observed in the SN Ia sample (excluding SNe Ia with no identified host). This correction is performed for separate redshift ranges and Deep fields to account for incompleteness in observations; values are given in Table 1. The result is the number of SN Ia expected in each galaxy over the observing period. These calculations are also repeated using the $A$ (mass) component only.

\section{PARAMETERIZED CLUSTER-LIKE ENVIRONMENTS}

To begin we use a continuous clustering strength estimator to compare the SN Ia rate per unit mass in and out of clustered environments. This parameter quantifies the significance $(\Sigma)$ of finding $N_{E}$ neighbor galaxies, the environment of a galaxy or SN Ia, given number expected from the background distribution. $\Sigma$ is calculated for a cylindrical volume environment of diameter $D$ and redshift depth $\pm \sigma_{\Delta z /(1+z)}(1+z)$ ( $\sigma$ from Section 2.4) centered on the object, as in Equation (1) where $N_{E}$ is the number of galaxies in the environment, $N_{F}$ is the number of galaxies within $\pm \sigma_{\Delta z /(1+z)}(1+z)$ in the Deep field, $A_{E}=\pi D^{2} / 4$ is the aperture area, and $A_{F}$ is the area of the field after allowing for the area occulted by foreground star masks (Ilbert et al. 2006). Since $N_{F}$ is computed at the same redshift as the object being studied, incompleteness at high redshift is automatically compensated for:

$$
\Sigma=\frac{N_{E}-N_{F}\left(A_{E} / A_{F}\right)}{\sqrt{N_{F}\left(A_{E} / A_{F}\right)}} .
$$

The advantage of this parametrization is that a strict cluster definition is avoided - any desired scale of clustering can be explored by altering the aperture diameter $D$. Environmental significances are calculated using a range of clustering scales (diameters $D$ ranging from 0.1 to $1.5 \mathrm{Mpc}$ ) both for field galaxies $(0.1<z<1.1)$, and also for the 232 SNe Ia surviving the cuts in Section 2.4. Normalized cumulative significance distributions for both field galaxies and SNe Ia are shown in Figure 1 for $D=0.6 \mathrm{Mpc}$. A Kolmogorov-Smirnov test shows that the two samples are statistically indistinguishable: SN hosts appear to be drawn from the same population as field galaxies with respect to clustering in their environment.

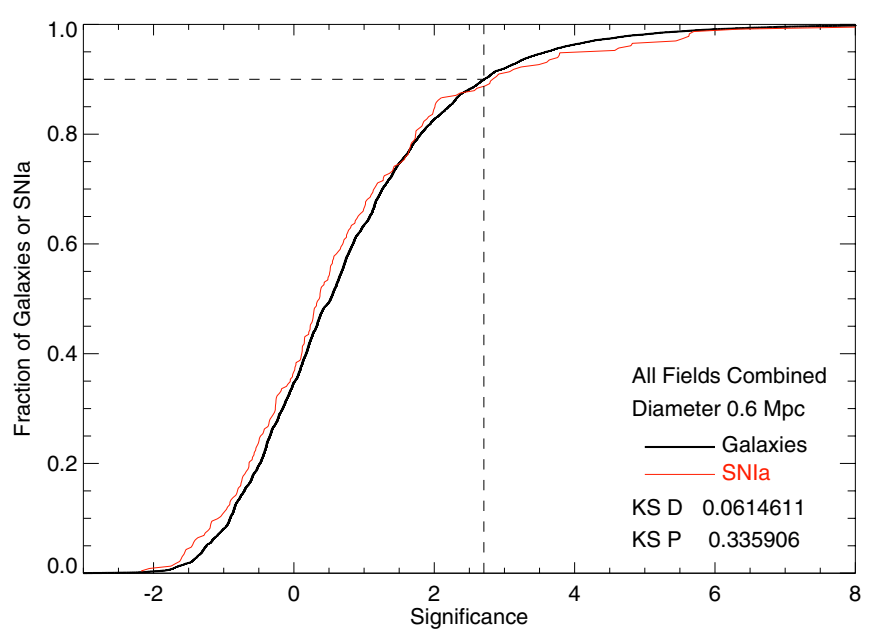

Figure 1. Cumulative significance distribution for galaxies and SNe Ia, with environment diameter $0.6 \mathrm{Mpc}$, for D1-4. Kolmogorov-Smirnov maximum difference (KS D) and probability (KS P) show the null hypothesis cannot be ruled out. Dashed lines represent $\Sigma_{10 \%}$.

(A color version of this figure is available in the online journal)

Let us now turn to the most clustered environments, by defining a significance limit $\left(\Sigma_{X \%}\right)$ to isolate the top $10 \%$, $5 \%$, and $1 \%$ most significant galaxy environments-the high significance group (HSG). Figure 1 shows the HSG cutoffs for $\Sigma_{10 \%}$ as an example; actually the HSG is determined for each diameter $D$ and each Deep field separately, then the HSGs for all are fields combined for a given $D$. We use summed Poisson probabilities to compare the number of SNe Ia in the HSG $\left(N_{\text {obs }}\right)$ to the number expected ( $\left.N_{\text {exp }}\right)$, which is the sum of the expected number of SNe Ia in each HSG galaxy (from Section 2.4). The Poisson probability of observing $x=N_{\text {obs }}$ given $\mu=N_{\exp }$ is expressed by Equation (2) (Bevington \& Robinson 2003), and the summed Poisson probabilities for $x>\mu$ and $x<\mu$ are given in Equations (3) and (4), respectively,

$$
\begin{gathered}
P_{P}(x ; \mu)=\mu^{x} \frac{e^{-\mu}}{x !} \\
P_{\mathrm{SUM}}(x, \infty ; \mu)=\sum_{x^{\prime}=x, x+1, \ldots}^{x^{\prime}=\infty} P_{P}\left(x^{\prime} ; \mu\right) \\
P_{\mathrm{SUM}}(0, x ; \mu)=\sum_{x^{\prime}=0,1, \ldots}^{x^{\prime}=x-1} P_{P}\left(x^{\prime} ; \mu\right) .
\end{gathered}
$$

As expressed by the morphology-density relation, galaxy clusters are dominated by early-type galaxies (Postman \& Geller 1984); so Poisson probabilities are also calculated for the subset of early-type field galaxies and SNe Ia hosts in the HSG. Results for a representative sample of environment diameters in Table 2 show the number of HSG SN Ia to be consistent with expectations of the two-component model over a range of $D$ and $\Sigma_{X \%}$. Thus we conclude that this clustering parametrization method does not show an influence of clustering on SN Ia events. Neither including the SNe Ia outliers rejected in Section 2.4, nor increasing the environment redshift depth to $\pm 2 \sigma(1+z)$, affects this conclusion.

\section{SN Ia IN OLSEN CATALOG CLUSTERS}

Here we identify SNe Ia and galaxies in grade A clusters from the Olsen et al. (2007) cluster catalog. We use Poisson 
Table 2

Results of the Clustering Parametrization Method for D1-4

\begin{tabular}{|c|c|c|c|c|c|c|c|c|c|}
\hline \multirow{2}{*}{$\begin{array}{l}\text { Diameter } \\
(\mathrm{Mpc})\end{array}$} & \multicolumn{3}{|c|}{$\Sigma_{10 \%}$} & \multicolumn{3}{|c|}{$\Sigma_{5 \%}$} & \multicolumn{3}{|c|}{$\Sigma_{1 \%}$} \\
\hline & $N_{\text {obs }}$ & $N_{\exp }$ & $P_{\text {SUM }}$ & $N_{\text {obs }}$ & $N_{\exp }$ & $P_{\text {SUM }}$ & $N_{\text {obs }}$ & $N_{\text {exp }}$ & $P_{\mathrm{SUM}}$ \\
\hline 0.4 & 21 & 26.9 & 0.15 & 11 & 15.0 & 0.18 & 3 & 3.98 & 0.44 \\
\hline 0.6 & 27 & 26.5 & 0.48 & 16 & 14.7 & 0.41 & 5 & 3.41 & 0.26 \\
\hline 0.8 & 21 & 25.3 & 0.23 & 12 & 13.8 & 0.38 & 5 & 3.15 & 0.21 \\
\hline 1.0 & 23 & 24.8 & 0.41 & 10 & 13.2 & 0.23 & 6 & 3.13 & 0.10 \\
\hline 1.5 & 21 & 22.3 & 0.44 & 11 & 12.0 & 0.46 & 2 & 2.98 & 0.43 \\
\hline \multicolumn{10}{|c|}{ For Early-Type Galaxies and SN Ia Hosts Only } \\
\hline 0.4 & 5 & 7.43 & 0.25 & 3 & 3.77 & 0.48 & 0 & 0.72 & 0.49 \\
\hline 0.6 & 6 & 6.86 & 0.47 & 3 & 3.27 & 0.59 & 0 & 0.60 & 0.55 \\
\hline 0.8 & 5 & 6.33 & 0.39 & 3 & 3.08 & 0.63 & 1 & 0.49 & 0.39 \\
\hline 1.0 & 3 & 5.98 & 0.15 & 3 & 2.86 & 0.54 & 1 & 0.59 & 0.45 \\
\hline 1.5 & 2 & 5.72 & 0.08 & 2 & 2.76 & 0.48 & 0 & 0.61 & 0.54 \\
\hline
\end{tabular}

Table 3

Cluster SNe Ia Details

\begin{tabular}{lccccccccc}
\hline \hline $\begin{array}{l}\text { SN Ia } \\
\text { SNLS ID }\end{array}$ & $\begin{array}{c}\text { SN Ia } \\
\text { R.A. }\end{array}$ & $\begin{array}{c}\text { SN Ia } \\
\text { Decl. }\end{array}$ & $\begin{array}{c}\text { SN Ia } \\
z_{\text {spec }}\end{array}$ & $\begin{array}{c}\text { SN Ia } \\
\text { stretch }\end{array}$ & $\begin{array}{c}\text { Host } \\
\text { type }\end{array}$ & $\begin{array}{c}\text { Host } \\
M_{V}\end{array}$ & $\begin{array}{c}\text { Host } \\
\text { offset }\end{array}$ & $\begin{array}{c}\text { Cluster } \\
z_{\text {phot }}\end{array}$ & $\begin{array}{c}\text { Cluster } \\
\text { offset }\end{array}$ \\
\hline 03D1ax & $02^{\mathrm{h}} 24^{\mathrm{m}} 23.32$ & $-04^{\circ} 43^{\prime} 14^{\prime \prime} .41$ & 0.496 & $\ldots$ & ES/0 & -23.64 & $1.97^{\prime \prime}$ & 0.53 & $10.8^{\prime \prime}$ \\
06D1kg & $02^{\mathrm{h}} 24^{\mathrm{m}} 32^{\mathrm{s}} .57$ & $-04^{\circ} 15^{\prime} 02^{\prime \prime} .0$ & 0.323 & 1.21 & Sbc & -20.31 & $1.69^{\prime \prime}$ & 0.30 & $47.2^{\prime \prime}$ \\
05D1by & $02^{\mathrm{h}} 24^{\mathrm{m}} 35^{\mathrm{s}} .45$ & $-04^{\circ} 12^{\prime} 04^{\prime \prime} .2$ & 0.299 & 0.99 & Sbc & -21.17 & $0.86^{\prime \prime}$ & 0.30 & $138.6^{\prime \prime}$ \\
04D1pg & $02^{\mathrm{h}} 27^{\mathrm{m}} 04^{\mathrm{s}} .16$ & $-04^{\circ} 10^{\prime} 31^{\prime \prime} .4$ & 0.515 & 1.05 & Sbc & -19.62 & $0.18^{\prime \prime}$ & 0.51 & $85.2^{\prime \prime}$ \\
05D3mq & $14^{\mathrm{h}} 19^{\mathrm{m}} 00^{\mathrm{s}} .40$ & $+52^{\circ} 23^{\prime} 06^{\prime \prime} .81$ & 0.246 & 0.90 & ES/0 & -21.85 & $4.94^{\prime \prime}$ & 0.25 & $9.99^{\prime \prime}$ \\
07D3af & $14^{\mathrm{h}} 19^{\mathrm{m}} 05^{\mathrm{s}} .01$ & $+53^{\circ} 06^{\prime} 08^{\prime \prime} .98$ & 0.356 & 0.98 & Scd & -18.61 & $0.35^{\prime \prime}$ & 0.30 & $128.7^{\prime \prime}$ \\
\hline
\end{tabular}

probabilities (Section 4.1) and a direct calculation of SNe Ia rates in clusters (Section 4.2) to look for an influence of clustering on the SN Ia rate per unit mass. As the SNLS detection efficiencies of Neill et al. (2006) are valid for $0.2<z<0.6$, we restrict cluster, galaxy, and SN Ia redshifts to this range to use of them. This decreases the catalogs to 30 clusters, 70,587 galaxies, and 109 SNe Ia. We note Olsen et al. (2007) and Ilbert et al. (2006) use different Terapix data releases (T0002 and T0003 respectively), and the release used by Olsen et al. has more masked regions. However, the differences in field effective areas are $\lesssim 10 \%$, so likely only $\lesssim 3$ clusters of Ilbert catalog galaxies are missing from the Olsen catalog.

$\mathrm{SNe}$ Ia and galaxy members of clusters are identified as are neighbors in the environment of a galaxy in Section 3, except the volume is centered on the cluster coordinates. Since the cluster filter used by Olsen et al. (2007) has a profile with a core radius of $r_{c}=0.133 h_{75}^{-1} \mathrm{Mpc}$ and a cut-off radius of $r_{\mathrm{co}}=1.33 h_{75}^{-1} \mathrm{Mpc}$, results for $0.4,0.8$, and $1.5 \mathrm{Mpc}$ will be presented as representative. For galaxies a redshift depth of $\pm 2 \sqrt{\left(\sigma_{\Delta z /\left(1+z_{\mathrm{SN}}\right)}\right)^{2}+\left(\sigma_{\Delta z /\left(1+z_{\mathrm{VVDS}}\right)}\right)^{2}}\left(1+z_{C}\right)$ is used, a convolution of uncertainties in cluster redshifts from Section 2.3 and galaxy redshifts from Section 2.4. As $\mathrm{SNe}$ Ia have spectroscopic redshifts, a redshift depth of simply $\pm 2 \sigma_{\Delta z /\left(1+z_{\mathrm{VVDS}}\right)}$ is appropriate. The images in Figure 2 and data in Table 3 present six SNe Ia identified in Olsen clusters. While the three SNe Ia within $D=0.8 \mathrm{Mpc}$ are probably physically associated with the clusters, this cannot be said for the remaining three. The number of field SNe Ia with $0.2<z_{\mathrm{SN}}<0.6$ predict $\sim 1.8 \mathrm{SNe}$ Ia would randomly appear in the regions between $r=0.4$ and $r=0.75 \mathrm{Mpc}$ and $\pm 2 \sigma_{\Delta z /\left(1+z_{\mathrm{VvDS}}\right)}$. The probabilities of all three being random and all three not being random associates are both $<20 \%$. As it remains likely that at least one is physically associated with a cluster, we include them in our results, but remind the reader that interloping SNe Ia (and galaxies) will add to the uncertainties for $D=1.5 \mathrm{Mpc}$.
Table 4

Summed Poisson Probabilities for Cluster SNe Ia for D1-4

\begin{tabular}{lcccccc}
\hline \hline Cluster & & \multicolumn{2}{c}{$A+B$ components } & & \multicolumn{2}{c}{$A$ component } \\
\cline { 3 - 4 } diameter & $N_{\text {obs }}$ & $N_{\text {exp }}$ & $P_{\text {SUM }}$ & & $N_{\text {exp }}$ & $P_{\text {SUM }}$ \\
\hline 0.4 & 2 & 2.38 & 0.57 & 3.21 & 0.38 \\
0.8 & 3 & 3.51 & 0.54 & 4.53 & 0.34 \\
1.5 & 6 & 5.97 & 0.55 & 7.30 & 0.41 \\
& 2 & For Early-Type Galaxies Only & & \\
0.4 & 2 & 1.97 & 0.59 & 2.79 & 0.47 \\
0.8 & 2 & 2.60 & 0.52 & 3.67 & 0.29 \\
1.5 & 2.69 & 0.29 & 5.23 & 0.11 \\
\hline
\end{tabular}

\subsection{Summed Poisson Probabilities}

Summed Poisson probabilities (defined in Section 3) of observing these cluster SNe Ia are computed from the number expected in the identified member galaxies, for all galaxy types and early-types only. Table 4 shows these observations are consistent with the two-component SN Ia rate model. In fact, $\geqslant 6 \mathrm{SNe}$ Ia would have to have been observed within $D=$ $0.4 \mathrm{Mpc}$ of galaxy clusters $(\geqslant 8$ or 0 for $D=0.8 \mathrm{Mpc})$ for $P_{\mathrm{SUM}}<0.05$. This constrains the $\mathrm{SN}$ Ia rate in clusters to agree with the two-component model to within a factor of 2 .

Obviously, to detect an effect on SNe Ia rates due to clustering (over and above the expectations of the two-component model and morphology-density relation) lurking in these data, we should at least detect the two-component model. So can we rule out the single component model (mass or " $A$ " component only) from these cluster observations? The answer appears to be no. Table 4 shows that the number of observed cluster SN Ia is actually consistent with both models. Surveys to test for the $A+B$ model or more complicated delay time distributions (Mannucci et al. 2006) will require a larger survey (two to three times the 

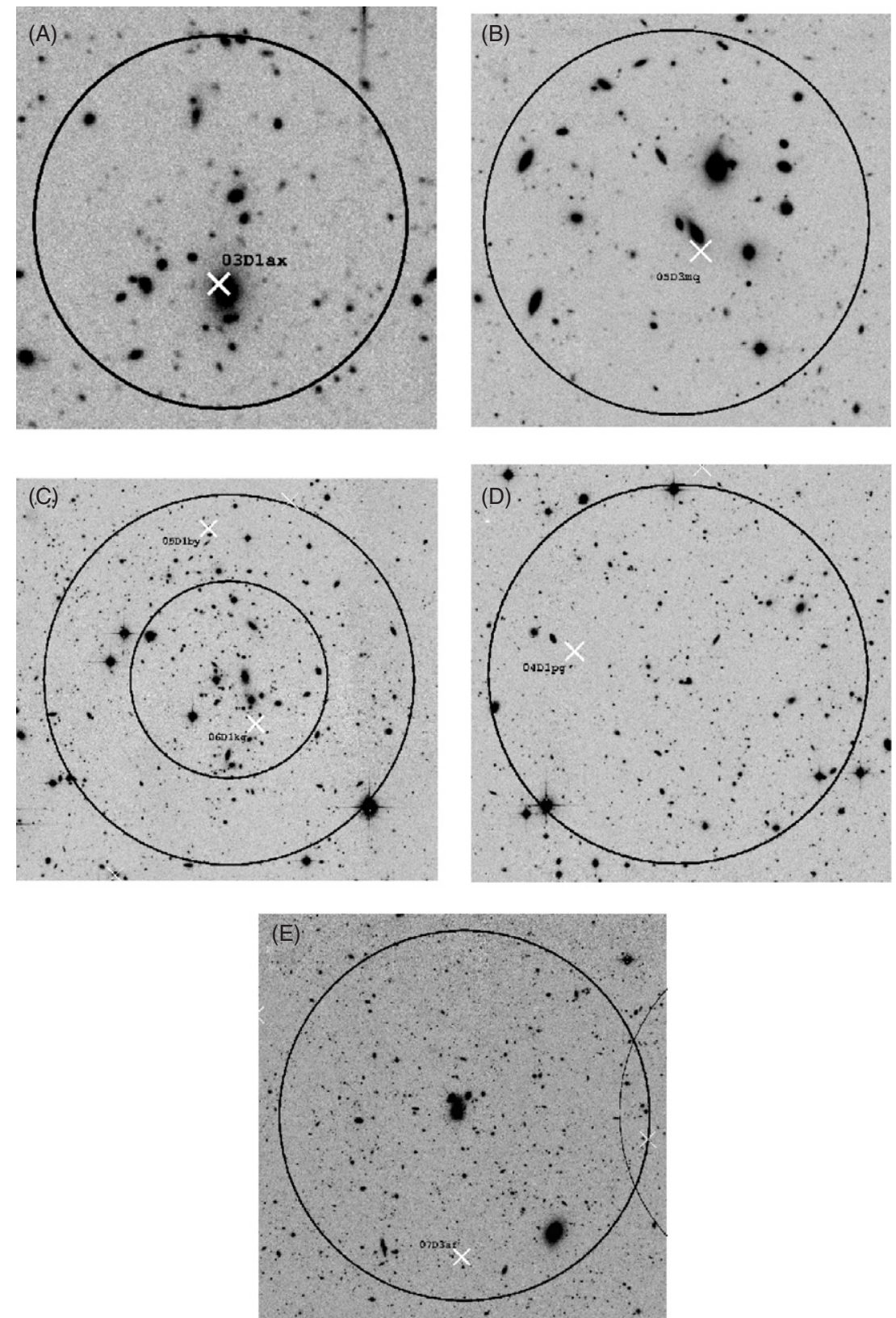

Figure 2. SNLS SNe Ia identified in galaxy clusters from the Olsen et al. (2007) catalog. SNe Ia coordinates marked by crosses, clusters represented by circles of diameter 0.4 Mpc (A and B), 0.8 Mpc (inner circle of C), and 1.5 Mpc (outer circle of C, D, and E). Images created from SNLS 2004 reference images and Skycat.

area or duration); unfortunately, the final SNLS data set will not be adequate for this.

\subsection{SN Ia Rates in Clusters}

Here we use detection efficiencies from Monte Carlo simulations of the SNLS SN Ia identification pipeline from Neill et al. (2006) to directly calculate the SN Ia rate per unit mass in clusters, and compare it to that for the field. The corrected number of SN Ia which exploded in a given field in a year, $N_{\text {corr, Ia }}$, is extracted from Equation (3) of Neill et al. (2006). As shown in Equation (5), $N_{\text {Ia }}$ is the total number of supernova observed in a given field, $S$ is the number of observing seasons, $\epsilon_{y r}$ is the detection efficiency per year (the probability of a supernova being detected, sent for spectroscopy, and identified as a type Ia), and $C_{\mathrm{SPEC}}$ accounts for the fraction of SNe Ia for which spectra are obtained yet remain unidentified. $\left[1+\langle z\rangle_{V}\right]$ corrects for time dilation at the volume-weighted average redshift of the survey, and $\langle z\rangle_{V}=0.46$. Detection efficiencies from Neill et al. (2006) and the number of observing seasons for each field up to 2007 January are in Table 5. Approximations to Poisson uncertainty from Gehrels (1986) determine the upper and lower limits on $N_{\text {Ia }}$ at the 0.84 confidence level (corresponding to $1 \sigma$ ), which are substituted into Equation (5) to determine $\Delta N_{\text {corr, Ia }}$ :

$$
N_{\text {corr,Ia }}=\frac{N_{\text {Ia }} / S}{\epsilon_{y r} C_{\mathrm{SPEC}}}\left[1+\langle z\rangle_{V}\right]
$$

Table 6 contains the resulting corrected SN Ia rate per unit mass per year in clusters and the field. With only three identified cluster SNe Ia, statistical uncertainties dominate this calculation; systematics, mainly the error in galaxy mass calculations, are likely another $\sim 30 \%$. Thus, our SN Ia rate is consistent with both 
Table 5

Parameter Values for Equation (5)

\begin{tabular}{lccc}
\hline \hline Field & Seasons & $\epsilon_{y r}{ }^{\mathrm{a}}$ & $C_{\text {SPEC }^{\mathrm{a}}}$ \\
\hline D1 & 4.0 & 0.3 & 0.94 \\
D2 & 4.0 & 0.22 & 0.88 \\
D3 & 4.0 & 0.31 & 0.80 \\
D4 & 4.1 & 0.31 & 0.69
\end{tabular}

Note. From Neill et al (2006).

the rate in early-type galaxies, $5.3 \pm 1.1 \times 10^{-14} \mathrm{SNe}^{-1} M_{\odot}^{-1}$ (Sullivan et al. 2006a), and the low-redshift cluster rate from WOOTS, $9.8_{-3.9}^{+5.9} \pm 0.9 \times 10^{-14} \mathrm{SNe} \mathrm{yr}^{-1} M_{\odot}^{-1}$ (Sharon et al. 2007).

There are two factors not considered which could affect SN Ia detection efficiencies in cluster galaxies. First, SN Ia detection efficiencies decrease in brighter hosts (Neill et al. 2006), and the brightest galaxies are early-type. Second, the SN Ia detection efficiency decreases for fainter, lower stretch SN Ia, and these faint SN Ia occur preferentially in earlytype hosts (Sullivan et al. 2006a). Since cluster galaxies are predominantly early-type, both of these effects dominate in clusters: the first effect decreases the number expected in clusters by $\sim 15 \%$, but quantifying the second would require more detailed completeness simulations. Both effects would cause us to underestimate the rate of $\mathrm{SNe}$ Ia in clusters relative to the field.

\subsection{SN Ia Rates in E/SO Cluster Galaxies}

To avoid these effects and the morphology-density relation, we limit the galaxy sample to two subsets: all early-type galaxies, and the brightest population of early-type galaxies (those with $M_{V}<-23.0$ like brightest cluster galaxies, BCGs). This has the added benefit of rejecting interlopers misidentified as cluster members. Two cluster SNe Ia have early-type hosts, and the host of 03D1ax is brighter than $M_{V}=-23.0$. Detection efficiency corrections are performed as described above, with the final results and rates presented in Table 6.

Although these samples are more sensitive to the two detection efficiency biases affecting early-type galaxies (Section 4.2), limiting all galaxies to early-types minimizes differences between the clusters and the field and results in a more meaningful test. The results, although not statistically significant, are suggestive of the rate enhancement in cluster over field early-type galaxies established by Mannucci et al. (2007). An enhancement in BCG-like galaxies would be consistent with the findings of an enhanced SN Ia rate in radio loud elliptical galaxies (Della Valle et al. 2005), as these are usually the brightest cluster galaxies. Future work will investigate the rates of SNLS SNe Ia in radio galaxies using existing radio catalogs for the Deep fields.

\subsection{Effects of Altering Data Constraints}

Including grade B clusters from the Olsen et al. (2007) catalog increases the total number of clusters to 65 , with one new cluster SN Ia identified (03D1fb at $z_{\mathrm{SN}}=0.498$ in an $\mathrm{E} / \mathrm{S} 0$ host of $M_{V}=-21.9$ with cluster offset $57.8^{\prime \prime}$ ). This does not affect the conclusions of the Poisson probability experiment (Section 4.1). The resulting cluster rates for $D=0.8 \mathrm{Mpc}$ for all galaxy types, early-types, and the brightest early-types only are $6.7_{-3.0}^{+8.5}, 6.7_{-3.4}^{+11.0}$, and $6.2_{-5.1}^{+29.2} \times 10^{-14} \mathrm{SNe} \mathrm{yr}^{-1} M_{\odot}^{-1}$; slightly below yet well within uncertainties of results with A grade clusters only.

None of the SNe Ia rejected as outliers in Section 2.4 are associated with Olsen catalog clusters. Including them does not affect the conclusions of the Poisson probability experiment, and only increases the $\mathrm{SNe}$ Ia field rate for all galaxy types to $15.9_{-1.5}^{+1.8} \times 10^{-14} \mathrm{SNe} \mathrm{yr}^{-1} M_{\odot}^{-1}$. Including in the experiment all SNe Ia for which no host was identified does not yield any new cluster SNe Ia either.

Reducing the SNe Ia sample to those included in Neill et al. (2006) avoids a possible over-correction for detection efficiencies which might arise from extending the sample to later times, if the survey became more efficient (we estimate any improvement in detection efficiency to be small). This decreases the number of SNe Ia to 40, with one cluster SNe Ia (03D1ax). This does not alter the overall conclusions of these experiments.

Lastly, the inclusion of SNLS SNe Ia candidates which did not receive spectroscopic confirmation (past maximum light, or detected as field season was ending), but which were given photometric types and redshifts using the techniques of Sullivan et al. (2006b), does not yield any new cluster SNe Ia or affect the conclusions of these experiments.

\section{CONCLUSIONS}

Type Ia supernova, galaxy, and cluster catalogs generated from the first four years of CFHT SNLS Deep survey data were

Table 6

Corrected SNe Ia Rates Per Unit Mass for D1-4

\begin{tabular}{|c|c|c|c|c|}
\hline $\begin{array}{l}\text { Galaxy } \\
\text { set }\end{array}$ & $N_{\text {Ia }}$ & $\begin{array}{c}N_{\text {corr,Ia }} \\
\left(\mathrm{SNe} \mathrm{yr}^{-1}\right)\end{array}$ & $\begin{array}{l}\text { Stellar mass } \\
\left(\times 10^{14} \mathrm{M}_{\odot}\right)\end{array}$ & $\begin{array}{c}\text { SN Ia rate } \\
\left(\times 10^{-14} \mathrm{SNe} \mathrm{yr}^{-1} \mathrm{M}_{\odot}^{-1}\right)\end{array}$ \\
\hline Clusters, $0.4 \mathrm{Mpc}$ & 2 & $2.8_{-1.6}^{+6.4}$ & 0.33 & $8.3_{-4.9}^{+19.4}$ \\
\hline Clusters, $0.8 \mathrm{Mpc}$ & 3 & $4.1_{-2.1}^{-1.6}$ & 0.48 & $8.5_{-4.3}^{+14.0}$ \\
\hline Clusters, $1.5 \mathrm{Mpc}$ & 6 & $8.1_{-3.1}^{+7.1}$ & 0.78 & $10.4_{-4.4}^{+9.3}$ \\
\hline Whole field & 109 & $169.1_{-16.3}^{+19.8}$ & 11.4 & $14.8_{-1.4}^{+1.7}$ \\
\hline \multicolumn{5}{|c|}{ For Early-Type Galaxies Only } \\
\hline Clusters, $0.4 \mathrm{Mpc}$ & 2 & $2.8_{-16}^{+6.4}$ & 0.29 & $9.6_{-5.6}^{+22.4}$ \\
\hline Clusters, $0.8 \mathrm{Mpc}$ & 2 & $2.8_{-16}^{+6.4}$ & 0.38 & $7.2_{-4.2}^{+16.0}$ \\
\hline Clusters, $1.5 \mathrm{Mpc}$ & 2 & $2.8^{+6.4}$ & 0.56 & $5.0^{+11.6}$ \\
\hline Whole field & 23 & $35.1_{-7.2}^{+11.0}$ & 6.23 & $5.6_{-1.2}^{+1.8}$ \\
\hline \multicolumn{5}{|c|}{ For the Brightest Early-Type Galaxies Only } \\
\hline Clusters, $0.4 \mathrm{Mpc}$ & 1 & $1.3_{-1.1}^{+6.1}$ & 0.13 & $9.8_{-8.1}^{+45.9}$ \\
\hline Clusters, $0.8 \mathrm{Mpc}$ & 1 & $1.3_{-1.1}^{+6.1}$ & 0.15 & $8.7_{-7.2}^{+40.9}$ \\
\hline Clusters, $1.5 \mathrm{Mpc}$ & 1 & $1.3_{-1}^{+6.1}$ & 0.19 & $7.0+33.0$ \\
\hline Whole field & 2 & $2.6_{-1.7}^{+6.3}$ & 1.02 & $2.5_{-1.6}^{+6.2}$ \\
\hline
\end{tabular}


combined to search for an influence of clustering on the SN Ia rate per unit mass. To avoid cluster-specific detection efficiencies and the inclusion of interloping galaxies as cluster members, we also considered subsets of regular and BCG-like early-type galaxies only. Results are dominated by the statistical uncertainties in identifying only three probable and three possible cluster $\mathrm{SNe}$ Ia, and consistent with the results of low-redshift cluster SNe Ia rate studies. To capitalize on SNLS's inclusion of both field and cluster environments, we used the continuous clustering strength parameter "significance" to compare the SN Ia rate per unit mass in and out of clustered environments, but did not find evidence of a clustering influence on the SN Ia rate per unit mass. Future work includes incorporating existing radio and infrared catalogs to investigate the rates of SNLS SNe Ia in radio loud and infrared luminous galaxies, and the VIRMOS spectroscopic redshifts for Deep field galaxies to explore the $\mathrm{SNe}$ Ia rate in small groups.

We gratefully acknowledge the CFHT Queued Service Observations team, Olivier Ilbert and Henry McCracken for early access to and correspondence regarding their photometric redshift galaxy catalog, Lisbeth Olsen for early access the optical cluster catalog, Jon Willis and Dan Maoz for helpful conversations, and our anonymous referee for constructive correspondence. This paper is based on observations obtained with MegaPrime/MegaCam, a joint project of CFHT and CEA/DAPNIA, at the CFHT, which is operated by the National Research Council of Canada, the Institut National des Science de l'Univers of the Centre National de la Recherche Scientifique (CNRS) of France, and the University of Hawaii. This paper is also based on spectroscopic observations obtained at the Gemini Observatory which is operated by the Association of Universities for Research in Astronomy, Inc., under a cooperative agreement with the NSF on behalf of the Gemini partnership; the Very Large Telescope at the European Southern Observatory in Paranal, Chile; the W. M. Keck Observatory which is operated as a scientific partnership among the California Institute of Technology, the University of California, and the National Aeronautics and Space Administration; and the 6.5 m Magellan Telescopes located at Las Campanas Observatory, Chile. This work is based in part on data products from the
Canadian Astronomy Data Centre as part of the CFHT Legacy Survey, a collaborative project of NRC and CNRS. This work has been supported by NSERC and the University of Victoria. Facilities: CFHT.

\section{REFERENCES}

Astier, P., et al. 2006, A\&A, 447, 31

Bevington, P., \& Robinson, R. 2003, in Data Reduction and Error Analysis for the Physical Sciences (New York: McGraw-Hill)

Buzzoni, A. 2005, MNRAS, 361, 725

Cappellaro, E., Evans, R., \& Turatto, M. 1999, A\&A, 351, 459

Coleman, G. D., Wu, C. C., \& Weedman, D. W. 1980, ApJS, 43, 393

Della Valle, M., Panagia, N., Padovani, P., Cappellaro, E., Mannucci, F., \& Turatto, M. 2005, ApJ, 629, 750

Gal-Yam, A., Maoz, D., \& Sharon, K. 2002, MNRAS, 332, 37

Gal-Yam, A., Maoz, D., Guhathakurta, P., \& Filippenko, A. V. 2008, ApJ, in press (arXiv:0711.0808)

Gehrels, N. 1986, ApJ, 303, 336

Hillebrandt, W., \& Niemeyer, J. C. 2000, ARA\&A, 38, 191

Höflich, P., Gerardy, C., \& Linder, E. 2003, LNP, 635, 203

Howell, D. A., et al. 2005, ApJ, 634, 1190

Ilbert, O., et al. 2006, A\&A, 457, 841

Kinney, A. L., Calzetti, D., Bohlin, R. C., McQuade, K., Storchi-Bergmann, T., \& Schmitt, H. R. 1996, ApJ, 467, 38

Madrid, J. P., Sparks, W. B., Ferguson, H. C., Livio, M., \& Macchetto, D. 2007, ApJ, 654L, 41

Mannucci, F., Della Valle, M., \& Panagia, N. 2006, MNRAS, 370, 733

Mannucci, F., Della Valle, M., Panagia, N., Cappellaro, E., Cresci, G., Maiolino, R., Petrosian, A., \& Turatto, M. 2005, A\&A, 433, 807

Mannucci, F., Maoz, D., Sharon, K., Botticella, M. T., Della Valle, M., Gal-Yam, A., \& Panagia, N. 2007, MNRAS, in press (arXiv:0710.1094)

Maoz, D., \& Gal-Yam, A. 2004, MNRAS, 347, 951

Mazzali, P. A., Röpke, F. K., Benetti, S., \& Hillebrandt, W. 2007, Science, 315,825

Neill, J. D., et al. 2006, AJ, 132, 1126

Olsen, L. F., et al. 2007, A\&A, 461, 810

Perlmutter, S., et al. 1999, ApJ, 517, 565

Postman, M., \& Geller, M. J. 1984, ApJ, 281, 95

Pritchet, C. J., Howell, D. A., \& Sullivan, M. 2008, Nature, submitted

Riess, A., et al. 1998, AJ, 116, 1009

Scannapieco, E., \& Bildsten, L. 2005, ApJ, 629, L85

Sharon, K., Gal-Yam, A., Maoz, D., Filippenko, A. V., \& Guhathakurta, P. 2007, ApJ, 660, 1165

Spergel, D. N., et al. 2007, ApJS, 170, 377

Sullivan, M., et al. 2006a, ApJ, 648, 868

Sullivan, M., et al. 2006b, AJ, 131, 960

Wood-Vasey, M., et al. 2007, ApJ, 666, 694 